\title{
SUBGROUPS OF INFINITE INDEX IN THE MODULAR GROUP
}

\author{
by W. W. STOTHERS
}

(Received 18 June, 1976)

The modular group $\Gamma$ is the group of integral bilinear transformations of the extended complex plane which preserve the upper half-plane. It has the presentation $\left\langle x, y: x^{2}=\right.$ $\left.y^{3}=1\right\rangle$, and the generators can be chosen so that $u=x y$ maps $z$ to $z+1$.

By the Kurosh subgroup theorem, a subgroup $H$ of $\Gamma$ is the free product of groups isomorphic to $C_{2}$, to $C_{3}$ and to $\mathbf{Z}$. Let the number of factors be $r, s, t$ respectively. Since we are interested in the case where $H$ has infinite index, we allow $r, s$ and $t$ to be $\infty$.

Regarding $H$ as a transformation group, the elements of $\mathbf{Q}$ fall into equivalence classes modulo $H$. In each class, the stabilizers are $H$-conjugate, and each is $\Gamma$-conjugate to a subgroup of $\langle u\rangle$. Let $h$ denote the number of classes, $h_{0}$ the number with non-trivial stabilizer, and $h_{\infty}=h-h_{0}$. If the stabilizer of a point is $\Gamma$-conjugate to $\left\langle u^{c}\right\rangle$ with $c>0$, the class has cusp-width $c$. Let $c(1), \ldots, c\left(h_{0}\right)$ be the widths of relevant classes.

When $H$ has finite index $n$, then $h_{\infty}=0$, and $r, s$ and $h$ are finite, with $h \geqslant 1$. There is an integer $g \geqslant 0$ such that

$$
n=3 r+4 s+6 h+12 g-12 .
$$

Also,

$$
n=c(1)+\ldots+c(h) .
$$

The group $H$ has standard presentation

$$
\begin{aligned}
H=\left\langle E_{1}, \ldots, E_{r}, F_{1}, \ldots, F_{s}, P_{1}, \ldots, P_{h}, A_{1}, B_{1}, \ldots, A_{\mathrm{g}}, B_{\mathrm{g}}:\right. \\
\left.E_{i}^{2}=F_{i}^{3}=\prod_{i=1}^{r} E_{i} \prod_{j=1}^{s} F_{j} \prod_{k=1}^{h} P_{k} \prod_{m=1}^{8}\left[A_{m}, B_{m}\right]=1\right\rangle .
\end{aligned}
$$

We can drop the last relation and one of the parabolic elements-the $P_{k}$-to obtain $H$ as a free product. Note that $t=h+2 g-1$, and that the number of hyperbolic generators-the $A_{m}$ and $B_{m}$-is even. These results are proved in Lehner [2].

In [4], Millington showed that when $h=1$ and $n, r, s$ and $g$ are non-negative integers satisfying (1) with $n \geqslant 1$, a corresponding subgroup exists. In [5], she extended this result to $h \geqslant 1$. In [7], some results for integers satisfying (2) are obtained.

In [8], Tretkoff showed that when $h=h_{\infty}=1$ and $r+s+t$ is infinite, with $t$ even if it is finite, a corresponding subgroup exists. We show that the parity restriction is necessary.

The method in [4], [5], and [8] is the exploitation of a correspondence between subgroups and permutations. The required permutations are built from basic patterns. Millington's patterns increase $r$ by $1, s$ by $1, g$ by 1 or $h$ by 1 . Tretkoff's patterns for $r$ and $s$ are identical. The pattern she uses to increase $t$ by 2 could be replaced by Millington's pattern for $\mathbf{g}$. The work in [4], [8] is simplified by the observation that all the cosets are of 
the form $H u^{k}$, so that $u$ induces the permutation $k \mapsto k+1(\bmod n)$. The permutation induced by $x$ is sufficient to identify the subgroup. In each case, the subgroup is recovered by the Reidemeister-Schreier process.

We use the method of coset diagrams and graph theoretic technique to extend Tretkoff's results.

1. Definitions. Coset diagrams were used in [6], but to allow investigation of group structure we describe them in a different way.

A diagram is a finite or countable set of points, with one point marked $O$, together with red, blue and green loops and edges such that

(a) each point has a loop, or is the end of one red line,

(b) each point has a directed blue loop, or is a vertex of one directed blue triangle,

(c) each point has a directed green loop, or is the beginning of one directed green line, and the end of another,

(d) the underlying graph is transitive,

(e) starting at any point, proceeding in positive direction when relevant, the walk 'red, blue' leads to the same point as the walk 'green'.

Given a diagram $D, D_{1}$ (resp. $D_{2}$ ) is the figure obtained by deleting the green (resp. blue) loops and edges from $D$.

Words of $\Gamma$ correspond to walks in $D$ starting from $O$. When words (in $x$ and $y$, or $x$ and $u$ ) are read from left to right, the walk is obtained by replacing $x$ by red, $y$ by blue and $u$ by green. Conditions (a), (b), (c) and (e) ensure that there is no ambiguity.

An excursion is a walk which consists of a sequence of lines, followed by the same sequence in reverse order.

A route is a closed walk starting from $O$ which does not involve any excursions.

Any closed walk starting from $O$ defines a unique route. Further, given routes $R_{1}$ and $R_{2}$, the walk $R_{1}$ then $R_{2}$ is closed and so defines a route $R_{1} R_{2}$. With this operation, the routes of a graph form a group $\mathscr{R}$.

The group resembles the cycle group in some respects, and can be obtained from a spanning tree in the usual way (see [1]). We state some straight-forward results; the proofs are easy.

Proposition 1.1. Given a set of edges without cycles in a connected graph, there is a spanning tree containing the given set.

Proposirion 1.2. Given a connected pseudo-graph, with a spanning tree $T$, the chords of $T$ together with loops yield a basis for the route group.

With a suitable convention regarding directed loops, this extends to our diagrams.

THEOREM 1.3. There is a 1-1 correspondence between diagrams and subgroups of $\Gamma$. If diagram $D$ corresponds to the subgroup $H$, then

(i) $r$ is the number of red loops in $D$,

(ii) $s$ is the number of blue loops in $D$,

(iii) $h$ is the number of green components in $D, h_{0}$ is the number of finite components. The cusp-widths are the sizes of the finite green polygons. 
Some of the routes of a diagram derive from relations in $\Gamma$, viz. $y^{3}=1$ corresponds to a route $W$, blue, blue, blue, $W^{-1}$, where $W$ is a walk starting at $O$, and similarly for $u=x y . \mathscr{R}(\Gamma)$ is the normal closure of these routes in $\mathscr{R}$.

THEOREM 1.4. $H \simeq \mathscr{R} / \mathscr{R}(\Gamma)$.

This is the graph theoretic analogue of the Reidemeister-Schreier process.

2. Presentation of subgroups. For a subgroup $H$, the corresponding $D_{1}$ leads to a presentation for $H$. Take the set of edges comprising two sides from each blue triangle, and extend it to a spanning tree $T_{1}$. The blue chords give elements of $\mathscr{R}(\Gamma)$, and can be ignored. The red chords and the loops of both colours give independent generators of $\mathscr{R}$. Those which come from blue loops have order 3 in $\mathscr{R} / \mathscr{R}(\Gamma)$. We have a presentation of $H$ as a free product, without recourse to Kurosh.

A presentation resembling (3) for finite index subgroups is also possible in our case.

Since we have a diagram with more than two points, a blue loop is joined by a red edge to a vertex of a blue triangle. We obtain a new diagram by replacing each blue loop (with its attached red edge) by a red loop at the vertex of the associated triangle. The green lines of the new diagram are those required to satisfy (e). The values of $h_{0}$ and $h_{\infty}$ are unchanged. Given a presentation for the new group, one is easily obtained for the original. Thus we may assume that we have $s=0$.

We begin with $D_{2}$, and consider the set of green edges. From each finite component, we omit one edge. The remaining set can be augmented with red edges to form a spanning tree $T_{2}$. Observe that any $k$ green components of $T_{2}$ are joined by at most $k-1$ red edges.

$\mathscr{R}$ is generated by loops of $D_{2}$ and chords of $T_{2}$. The green loops and chords give rise to parabolic elements of $H . \mathscr{R}(\Gamma)$ consists (essentially) of the routes derived from the blue triangles of $D$.

With $D$, we associate a further graph $G$. In $D_{1}$ we remove the (red) loops, and all red edges which belong to $T_{2}$. Further, we replace each blue triangle by a vertex incident with all edges remaining on the triangle.

\section{LEMMA 2.1. G has no finite components.}

Proof. Let $C$ be a finite component of $G$, and let $\bar{C}$ be the corresponding portion of $D$, including all vertices of the corresponding blue triangles.

As $D$ is connected, $\bar{C}$ is attached to the rest of $D$ by $k(>0)$ red edges. Since $C$ is a component of $G$, all of these edges belong to $T_{2}$.

At each such edge, one green component of $D$ enters $\bar{C}$, and one, not necessarily distinct, leaves. As $C$ is finite, a green component which enters $C$ must leave, also at a red $T_{2}$ edge. Thus, the $k T_{2}$ edges link at most $k$ green components. This contradicts the observation made after the definition of $T_{2}$. 
LEMMA 2.2. Some of the edges of $G$ can be directed so that

(i) each vertex has one edge directed towards it,

(ii) each component of the directed subgraph is a tree,

(iii) the branch directed away from any point is finite.

Proof. The components of $G$ are infinite by 2.1. Each is dealt with in the same way.

Let $S$ be a spanning tree of one component, and let $P$ be a vertex of $S$. We proceed by stages; at the $k$ th stage we consider vertices at distance $k$ from $P$, including $k=0$.

Suppose that we have reached the point $Q$. The edges between $P$ and $Q$ have been considered, and we deal with the others as follows:

Case 1 , the edge belongs to a finite branch from $Q$, then direct the edge away from $Q$;

Case 2, the edge belongs to an infinite branch, then, if it is the first such edge encountered, direct it towards $Q$, otherwise delete it.

Since $S$ is infinite, each point receives an edge directed towards it. Since the directed subgraph is a subset of a forest, each of its components is a tree. By the process of construction, the edges directed away from a point belong either to a finite branch of the corresponding tree, or to the part of the tree between the point and the starting point of the relevant part of $G$. Hence, the third clause of the lemma holds.

THEOREM 2.3. If $H$ is a subgroup of infinite index in $\Gamma$, then it has the presentation

$$
H=\left\langle E_{1}, \ldots, E_{r}, F_{1}, \ldots, F_{s}, P_{1}, \ldots, P_{h_{0}}, A_{1}, \ldots, A_{m}: E_{i}^{2}=F_{i}^{3}=1\right\rangle,
$$

with $P_{i}$ parabolic, $A_{j}$ hyperbolic, and $t=h_{0}+m$.

Proof. As before, we assume that there are no blue loops in $D$. In the tree $T_{2}$ the green loops and chords give parabolic elements $P_{1}, \ldots, P_{h_{0}}$, the red loops give elliptic elements, and the red chords give hyperbolic elements $B_{1}, B_{2}, \ldots$

From a blue triangle in $D$ we obtain a relation (in $H$ )

$$
B_{a}^{\alpha} P_{b}^{\beta} B_{c}^{\gamma} P_{d}^{\delta} B_{e}^{\varepsilon} P_{f}^{\zeta}=1,
$$

where $\alpha, \ldots, \zeta$ are \pm 1 , and a $B$ or $P$ is omitted if the corresponding line is in $T_{2}$. Observe that if we have a triangle involving the same red edge twice, then the edge joins two vertices of the triangle and so there is a green loop at one vertex. As $T_{2}$ spans $D_{2}$, the red edge must belong to $T_{2}$. Thus any $B$ can appear only once in (4).

A blue triangle corresponds to a vertex in $G$, and the edge directed towards it in $G$ must correspond to a $B$ term in (4). As the exponent is \pm 1 , (4) can be rewritten to express this $B$ in terms of the other elements. Observe that any other directed $G$-edges involved are directed away from the vertex.

Let $\left\{B_{i}\right\}$ be the subset of $\left\{B_{i}\right\}$ corresponding to directed edges of $G$. Each blue triangle is associated with an element of the subset, and using the relations like (4), we can express the element in terms of the $P_{i}, B_{j}$ not in the subset, and $B_{k}$ belonging to the branch directed away from the triangle. By 2.2 (iii), there is a finite expression obtained by eliminating the last type of elements. 
Since all the relations stem from blue triangles, this process leads to the conclusion that we can remove the $B_{i^{\prime}}$, to get a set of generators of $H$ as a free product which contains a maximal set of parabolics, i.e. one for each finite green component.

This method can be used for subgroups of finite index, though in this case 2.1 and 2.2 require some alteration.

This leads to a presentation with a single relation. Some further manipulation is needed to obtain the standard presentation (3). As a corollary, we find that the parabolic generators are all $\Gamma$-conjugate to positive, or all to negative, powers of $u$. A geometrical proof of this is to be found in Mason [3].

A standard set for a subgroup of $\Gamma$ is a set of generators of the subgroup as a free product with the number of parabolic generators equal to $h_{0}$ if $n=\infty$, to $h_{n}-1$ if $n<\infty$.

Let $t_{1}$ be the number of hyperbolic elements in a standard set. Thus, in the usual notation,

$$
t= \begin{cases}t_{1}+h_{0}, & \text { if } n=\infty, \\ t_{1}+h_{0}-1, & \text { if } n<\infty .\end{cases}
$$

Lemma 2.4. For a diagram $D, t$ is equal to the number of red edges in $D_{1}-T_{1}$.

This is an obvious consequence of remarks in the first paragraph of this section.

LEMMA 2.5. For a diagram $D, t_{1}$ is equal to the number of red edges which do not belong to $T_{2}$ and do not correspond to directed edges of $G$.

3. Joining, cutting and composition. If we have two vertices each with a red loop, either in one or two diagrams, then we can obtain a new diagram by removing the loops and joining the vertices by a red edge. This is described as joining the vertices (or joining the diagrams). This is described in [6]. The basic patterns of Millington and of Tretkoff correspond to diagrams having two red loops. Their results are obtained by joining basic diagrams to form a chain.

We shall also use the idea of composition, see [6]. This is possible when we have two triangles each with at least two red loops attached, and can be viewed as two joins.

The operation of joining can be reversed, i.e. we can replace a red edge by two red loops. This process is described as cutting the edge. It should be noted that cutting may disconnect the diagram, and must do if the edge was involved in no cycle of the original $D_{1}$.

All of these operations involve changes in green loops or edges, and it is best to regard them as operations on $D_{1}$ diagrams with the green structure recovered using (e). Only two green lines are affected in any case, and it is easy to draw parts of diagrams to explain the changes. In $3.1,3.2$ we assume $D^{\prime} \neq D^{\prime \prime}$.

LemMa 3.1. Let $D^{\prime}$ (resp. $D^{\prime \prime}$ ) be a diagram with a red loop at $A$ (resp. B) and let $D$ be the diagram obtained by joining $A$ and $B$. Then, in an obvious notation, $n=n^{\prime}+n^{\prime \prime}$, $s=s^{\prime}+s^{\prime \prime}, r=r^{\prime}+r^{\prime \prime}-2, t=t^{\prime}+t^{\prime \prime}$. 
Proof. The first two equalities are obvious, since vertices and blue loops are unaltered.

Two red loops are used in joining, so the third holds.

If $T_{1}^{\prime}$ (resp. $T_{1}^{\prime \prime}$ ) is the usual tree in $D_{1}^{\prime}$ (resp. $D_{1}^{\prime \prime}$ ), then $T=\{A B\} \cup T_{1}^{\prime} \cup T_{1}^{\prime}$ is the corresponding tree in $D_{1}$. The fourth equality follows by 2.4 .

Lemma 3.2. With the notation of 3.1 , suppose that the green cycle in $D^{\prime}$ (resp. $D^{\prime \prime}$ ) which contains $A$ (resp. B) has length $a$ (resp. b). Then, in $D, h_{\infty}=h_{\infty}^{\prime}+h_{\infty}^{\prime \prime}$ and

(i) if $a$ or $b$ is finite, then $A$ and $B$ belong to $a$ green cycle of length $a+b$ and $h_{0}=h_{0}^{\prime}+h_{0}^{\prime \prime}-1$,

(ii) if $a=b=\infty$, then $A$ and $B$ belong to distinct infinite green cycles, and $h_{0}=h_{0}^{\prime}+h_{0}^{\prime \prime}$.

A sketch showing the green cycles involving $A$ and $B$ before and after joining gives the lengths of the cycles in $D$. The results for $h_{0}$ follow at once.

Corollary 3.3. In the notation of $3.1,3.2$,

(i) if either $D^{\prime}$ or $D^{\prime \prime}$ is finite, then $t_{1}=t_{1}^{\prime}+t_{1}^{\prime \prime}$,

(ii) if $a=b=\infty$, then $t_{1}=t_{1}^{\prime}+t_{1}^{\prime \prime}$.

Proof. (i) In this case, we must have the situation of 3.2 (i), so that $h_{0}=h_{0}^{\prime}+h_{0}^{\prime \prime}-1$. Since $D$ will be finite if and only if both $D^{\prime}$ and $D^{\prime \prime}$ are, the result follows by applying (5) and 3.1 .

LeMma 3.4. Let $D^{\prime}$ be a diagram with red loops at $A$ and $B$, and let $D$ be the result of joining $A$ and $B$. Then $n=n^{\prime}, s=s^{\prime}, r=r^{\prime}-2, t=t^{\prime}+1$.

LEMMA 3.5. In the notation of 3.4, if $A$ and $B$ belong to a single green cycle of length $a$ in $D^{\prime}$, then they belong to distinct green cycles of lengths $b, a-b$, with $b$ finite.

CoROllary 3.6. In the notation of $3.4,3.5, h_{\infty}=h_{\infty}^{\prime}, h_{0}=h_{0}^{\prime}+1, t_{1}=t_{1}^{\prime}$.

LEMMA 3.7. In the notation of 3.4, if $A$ and $B$ belong to distinct green cycles, of lengths $a$ and $b$ respectively, in $D^{\prime}$, then, in $D, h_{\infty}=h_{\infty}^{\prime}$, and

(i) if $a$ or $b$ is finite, then $A$ and $B$ belong to a green cycle of length $a+b$,

(ii) if $a=b=\infty$, then $A$ and $B$ belong to distinct, infinite, green cycles.

Corollary 3.8. In the notation of $3.4,3.7$,

(i) if $a$ or $b$ is finite, then $h_{0}=h_{0}^{\prime}-1, t_{1}=t_{1}^{\prime}+2$,

(ii) if $a=b=\infty$, then $h_{0}=h_{0}^{\prime}, t_{1}=t_{1}^{\prime}+1$.

In all cases, the proofs are similar to those of $3.1,3.2,3.3$.

Since cutting reverses joining, all of these results can be adapted to give results about cutting, see the proof of 4.4 .

These results could also be used to obtain results about composition, but if we recall (from [6]) that composition does not affect the length of green cycles, or their number, a more direct approach is possible. 
Lemma 3.9. If $D^{\prime}$ and $D^{\prime \prime}$ are $F(1)$ diagrams, then their composition $D$ has $n=n^{\prime}+n^{\prime \prime}$, $s=s^{\prime}+s^{\prime \prime}, r=r^{\prime}+r^{\prime \prime}-4, h_{0}=h_{0}^{\prime}+h_{0}^{\prime \prime}, h_{\infty}=h_{\infty}^{\prime}+h_{\infty}^{\prime \prime}, t=t^{\prime}+t^{\prime \prime}+1$. (See [6] for the definition of $F(1)$.)

Proof. The first five equalities are trivial. For the last, we observe that two red edges are added, but that only one is required to make trees for $D^{\prime}$ and $D^{\prime \prime}$ into a tree for $D$, and apply 2.4 .

As usual, (5) gives a corollary.

COROLlary 3.10. In the notation of 3.9,

(i) if $D^{\prime}$ or $D^{\prime \prime}$ is finite, then $t_{1}=t_{1}^{\prime}+t_{1}^{\prime \prime}$,

(ii) if $D^{\prime}$ and $D^{\prime \prime}$ are infinite, then $t_{1}=t_{1}^{\prime}+t_{1}^{\prime \prime}+1$.

Lemma 3.11. Let I be a finite or countable set. All sequences are indexed by 1 . Suppose that $D^{\prime}$ is a diagram with red loops at the vertices $\{A(i)\}$, and that $\{D(i)\}$ is a collection of diagrams, $D(i)$ having a red loop at $B(i), h_{0}=a(i), t_{1}=b(i), h_{\infty}=c(i)$. Let $D$ be the diagram obtained by joining $A(i)$ and $B(i)$, for each $i \in I$.

(i) If each $D(i)$ is finite, and no two $A(i)$ belong to the same finite green cycle of $D^{\prime}$, then $h_{\infty}=h_{\infty}^{\prime}, h_{0}=h_{0}^{\prime}+\sum(a(i)-1), t_{1}=t_{1}^{\prime}+\sum b(i)$.

(ii) If the $A(i)$ and $B(i)$ all belong to infinite green cycles in $D^{\prime}$ and $D(i)$, then $h_{0}=h_{0}^{\prime}+\sum a(i), t_{1}=t_{1}^{\prime}+\sum b(i), h_{\infty}=h_{\infty}^{\prime}+\sum c(i)$.

Proof. The statements about the number of green cycles are clear.

Let $O$ be the origin for $D^{\prime}$. There is a standard set of generators of the form $\bigcup_{i \in I}\left(\left\{c(i)^{-1} x c(i)\right\} \cup\{P(i)\} \cup E\right)$, where $c(i)$ corresponds to a walk from $A(i)$ to $O$, and $P(i)$ corresponds to the green cycle including $A(i)$ when this cycle is finite. The set $E$ includes the $t_{1}^{\prime}$ hyperbolic generators.

(i) Take $B(i)$ as the origin for $D(i)$. A standard set of generators (excluding the parabolic element corresponding to the green cycle through $B(i))$ has the form $\{x\} \cup E(i)$. The set $E(i)$ includes the $b(i)$ hyperbolic generators.

From the diagram $D$, it is obvious that, with $O$ as origin, a standard set is $\bigcup_{i \in I}\left(\{Q(i)\} \cup E \cup c(i)^{-1} x E(i) x c(i)\right)$, where $Q(i)$ corresponds to the green cycle in $D$ which includes $A(i)$ and $B(i)$, and so is present when the cycle in $D^{\prime}$ which includes $A(i)$ is finite, i.e. precisely when $P(i)$ is present. The $t_{1}$ result follows since the conjugate of a hyperbolic element is hyperbolic.

(ii) In this case, each $D(i)$ must be infinite, so that there is no need to exclude an element corresponding to one cycle. Also, the set for $D^{\prime}$ contains no $P(i)$. Otherwise, the proof is as for (i).

The proof of 3.11 indicates that it is quite possible to obtain standard sets of generators for a diagram obtained by joining separate diagrams. In the proof of 4.3 below, 
no attempt is made to produce such a set, but it should be clear that it could be obtained quite easily.

4. Existence results. For constructive proofs of various results, we use some small diagrams. In each case we give a description of the $D_{1}$ of the diagram, a standard set of generators and any useful facts.

$S$ : one vertex with red and blue loops, $\langle x, y\rangle$.

$R$ : three vertices with one blue triangle and three red loops, $\left\langle x, y x y^{2}, y^{2} x y\right\rangle$.

$T$ : nine vertices with three blue triangles, one red loop and four red edges, $\left\langle x, u^{-8} x u^{5}, u^{-2} x u^{6}\right\rangle$. (In this case, several diagrams are possible, but only one has $h_{0}=1$. We choose this as $T$, it has $t_{1}=2$.)

$P$ : three vertices with one blue triangle, one red loop and one red edge, $\left\langle x, y^{2} x y^{2}\right\rangle$. This has $h_{0}=2, c(1)=2, c(2)=1$.

Observe that joining a copy of $S$ to a diagram raises $n$ by $1, r$ by $-1, s$ by 1 , leaving others unchanged, by $3.1,3.2$ (i), 3.3 (i). Joining a copy of $T$ increases $t_{1}$ by 2 , and so, in a finite context, raises the genus $g$ by 1 , since $g=\frac{1}{2} t_{1}$. Joining a copy of $P$ increases $h_{0}$ by 1 . The diagram $R$ has a different purpose.

THEOREM 4.1. (Millington). Given a set of integers satisfying (1), with $n, h \geqslant 1$, and $r, s, g \geqslant 0$, there is a corresponding subgroup of $\Gamma$.

Proof. Let $m=r+s+g+h$. From (1), $m \geqslant 2$.

If $m \geqslant 4$, we take $m-3$ copies of $R$ and join them to form a chain. This is a diagram with $s=g=0, h=1, r=m-1$ (consider the $D_{1}$ and $T_{1}$ ). Using the red loops of the chain, we join to it $s$ copies of $S, h-1$ of $P$ and $g$ of $T$. A finite number of applications of 3.1, 3.2 (i) and 3.3 (i) show that the result is of the required type.

If $m=3$, one or two copies of $S, P$ and $T$ cover all cases.

If $m=2$, only $r=s=0, h=g=1$ give $n \geqslant 1$. There are three six vertex diagrams with $r=s=0$, and one of these is of the required type.

For subgroups of infinite index, some further diagrams are useful.

$C$ : a chain of copies of $R$, joined and extending indefinitely to the right. The $D_{1}$ and $T_{1}$ are obvious, and show that $r=\infty, s=t=0$, so that $h_{0}=t_{1}=0$. Also, $h_{\infty}=1$. Since the left-most triangle has two red loops, $C$ is $\mathrm{F}(1)$.

$R^{*}$ : the degenerate diagram with no vertices. If $E$ is a proper diagram, with a red loop at $A$, we "join" $R^{*}$ to $E$ by leaving $E$ alone but marking the red loop at $A$ to signify that it is not to be used for further joining.

$R_{1}$ : a chain of three copies of $R$ joined. This has $h_{0}=1, t_{1}=s=0$ and $r=5$. Further, $R_{1}$ is $\mathrm{F}(2)$, (see [6]), with a spare red loop.

$C_{1}$ : a chain of copies of $R_{1}$ extending indefinitely to the right, formed by composition. Since each vertex is in a green 9-cycle, $C_{1}$ has $h_{\infty}=0$ and $h_{0}=\infty$. Since $R_{1}$ has no blue loops and a red loop on the central triangle, $C_{1}$ has $s=0, r=\infty$. The red edges in the $T_{2}$ of $C_{1}$ consist of one from each pair introduced by 
composition. Thus, each red edge belongs to $T_{2}$ or corresponds to a directed edge of $G$. By $2.5, C_{1}$ has $t_{1}=0$.

$C_{1}^{+}$: a copy of $R$ composed with a copy of $C_{1}$. This has $h_{\infty}=0, h_{0}=\infty, s=0, r=\infty$, $t_{1}=0$.

$C_{2}$ : the composition of two copies of $C_{1}$. By 3.10 (ii), this has $t_{1}=1$, and, by 3.9, $s=h_{\infty}=0, h_{0}=r=\infty$. Note that $t_{1} \neq 0$, although this might be suggested by a simple-minded application of 3.10 (i) to infinitely many copies of $R_{1}$.

$C_{3}$ : the composition of a copy of $C$ with a copy of $C_{1}$. By 3.9 and 3.10 (ii), this has $s=0, h_{\infty}=1, h_{0}=r=\infty, t_{1}=1$.

$C_{4}$ : the composition of two copies of $C$. By 3.9 and 3.10 (ii), this has $h_{0}=s=0$, $h_{\infty}=2, r=\infty, t_{1}=1$.

$X$ : we form a sequence $\left\{X_{i}\right\}$, taking $X_{1}=R$ with one distinguished red loop. $X_{i+1}$ is formed from $X_{i}$ by joining a copy of $R$ to each non-distinguished red loop. The diagram $X$ is the "limit" of the sequence in the obvious sense. Using the $T_{1}$ and $D_{1}$ of $X$, it is clear that it has $r=1, s=h_{0}=t_{1}=0$ and $h_{\infty}=\infty$, see 4.2 below.

We note that, taking the red loop at the origin of $X$, the corresponding subgroup is $\langle x\rangle$.

Lemma 4.2. For a subgroup of infinite index in $\Gamma$,

$$
r+s+t_{1}+h_{0}+h_{\infty}=\infty \text {. }
$$

Proof. Suppose that we have a subgroup of infinite index in $\Gamma$ with $r+s+t$ finite. The corresponding $D_{1}$ has finitely many finite cycles apart from blue triangles. These involve a finite set of blue triangles, so that there is a finite connected portion containing these and all loops. The rest of the diagram has no loops or non-trivial cycles, so, here, each triangle has vertices in three distinct infinite green components. Moreover, no two triangles have more than one green component in common. As there are infinitely many triangles, $h_{\infty}=\infty$.

At several points in the next theorem, we shall have to consider the situation in 3.11 . As all diagrams are countable at worst, we shall assume that the red loops of the initial diagram $\left(D^{\prime}\right.$ in 3.11$)$, and the collection of other diagrams $(D(i)$ in 3.11) come with a numbering, and the other diagrams with a designated red loop.

THEOREM 4.3. Suppose that $r, s, h_{0}, h_{\infty}, t_{1} \geqslant 0$, with $r+s+h_{0}+h_{\infty}+t_{1}=\infty$. Then there is a corresponding subgroup of $\Gamma$ if any of the following conditions is satisfied:

(i) $h_{\infty}=0$,

(ii) $h_{\infty}=1$, and $t_{1}$ even if $t_{1}+h_{0}$ is finite,

(iii) $h_{\infty} \geqslant 2$.

Proof. (i) Suppose firstly that $t_{1}$ is even or finite. We take a copy of $C_{1}^{+}$, and a collection of $r$ copies of $R^{*}, s$ of $S, h_{0}$ of $P$ and $\frac{1}{2} t_{1}$ of $T$, with the obvious interpretation if $t_{1}$ is infinite. By 4.2 , this collection must be infinite since $h_{\infty}=0$. We join these diagrams as in 3.11 (i). The values of $r$ and $s$ are clearly as required, as are those of $h_{0}, h_{\infty}$ and $t_{1}$ by the lemma. 
Suppose now that $t_{1}$ is odd. We take a copy of $C_{2}, r$ copies of $R^{*}, s$ of $S, h_{0}$ of $P$ and $\frac{1}{2}\left(t_{1}-1\right)$ of $T$. We then proceed as above.

(ii) If $t_{1}$ is even or infinite, we can proceed as in first part of (i), using $C$ as initial diagram instead of $C_{1}^{+}$.

If $t_{1}$ is odd and $h_{0}=\infty$, then we proceed as in the second part of (i), using $C_{3}$ as initial diagram instead of $C_{2}$.

(iii) Suppose firstly that $t_{1}$ is even or infinite and $r+s+h_{0}+t_{1}$ infinite. We take a copy of $C$ and a collection of $\left(h_{\infty}-1\right)$ further copies of $C$. We join these to produce $C_{5}$, which, by 3.11 (ii), has correct value of $h_{\infty}$, and $s=h_{0}=t_{1}=0, r=\infty$. We proceed as in the first part of (i), using $C_{5}$ as initial diagram.

Suppose now that $t_{1}$ is odd and $r+s+h_{0}+t_{1}$ infinite. We proceed as in the previous paragraph, replacing the first copy of $C$ in the formation of the initial diagram by $C_{4}$.

Suppose finally that $r+s+h_{0}+t_{1}$ is finite. Then, by $4.2, h_{\infty}$ must be infinite. Using the techniques of the other parts of (iii), we can construct $C_{6}$, a diagram with required values of $s, h_{0}, t_{1}$, but with $h_{\infty}=2, r=\infty$. We join a copy of $X$ to all but $r$ of the red loops of $C_{6}$. By 3.11 (ii), this final diagram is as required.

Tretkoff's Theorem 2 is the special case of 4.3 (ii) with $h_{0}=0, r \geqslant 1$. Our last theorem shows that the parity condition of her theorem, and of 4.3 , is necessary.

THEOREM 4.4. There is no subgroup of $\Gamma$ with $h_{\infty}=1, h_{0}+t_{1}$ finite and $t_{1}$ odd.

Proof. Suppose that the diagram $D$ with $h_{\infty}=1, h_{0}+t_{1}$ finite. A corresponding standard set of generators has a finite number (viz. $h_{0}+t_{1}$ ) of non-elliptic elements. We can choose a finite, connected subgraph $D_{0}$ of $D_{1}$ which includes the routes corresponding to these generators, and complete blue triangles.

If a vertex of $D_{0}$ is linked to a vertex of $D_{1}-D_{0}$ by $c$, a red edge of $D$, then both of these vertices belong to the infinite green cycle. Since $c$ does not belong to any cycle of $D_{1}$, its removal must disconnect the diagram. Hence, if we cut $c$, the diagram splits into two. The component which does not contain $D_{0}$ has no non-trivial cycles, and so has $t_{1}=0$ and $h_{0}+h_{\infty}=1$. The cut must be the reverse of the situation described in 3.2 (i) and 3.3 (i). Thus, the part containing $D_{0}$ has the same value of $t_{1}$ as the original diagram.

We can repeat this process until all the red edges leaving $D_{0}$ have been cut. We are left with $D_{0}$, with some extra red loops. Since $D_{0}$ is finite, this final diagram is finite, so that its $t_{1}$ is even (see the remarks following (3)). Since this is the same as the value for the original diagram, the result follows.

I should like to thank the referee for some helpful suggestions regarding some of the proofs, particularly those of 2.1 and 4.2 .

\section{REFERENCES}

1. F. Harary, Graph theory (Addison-Wesley, 1969).

2. J. Lehner, Discontinuous groups and automorphic functions (Amer. Math. Soc., 1964).

3. A. W. Mason, On a theorem by Leon Greenberg, Proc. Amer. Math. Soc. 23 (1969), 18-23. 
4. M. H. Millington, On cycloidal subgroups of the modular group, Proc. London Math. Soc. (3), 19 (1969), 164-176.

5. M. H. Millington, Subgroups of the classical modular group, J. London Math Soc. (2), 1 (1969), 351-357.

6. W. W. Stothers, Subgroups of the modular group, Proc. Cambridge Philos. Soc. 75 (1974), 139-153.

7. W. W. Stothers, Impossible specifications for the modular group, Manuscripta Math. 13 (1974), 415-428.

8. C. Tretkoff, Nonparabolic subgroups of the modular group, Glasgow Math. J. 16 (1975), 91-102.

UNIVERSITY OF GLASGOW

Glasgow

G12 8QW 